\title{
Annamalai's Computing Model for Algorithmic Geometric Series and Its Mathematical Structures
}

\author{
Chinnaraji Annamalai \\ Vinod Gupta School of Management, Indian Institute of Technology, Kharagpur, India
}

\section{Email address:}

anna@iitkgp.ac.in

\section{To cite this article:}

Chinnaraji Annamalai. Annamalai's Computing Model for Algorithmic Geometric Series and Its Mathematical Structures. Mathematics and Computer Science. Vol. 3, No. 1, 2018, pp. 1-6. doi: 10.11648/j.mcs.20180301.11

Received: October 6, 2017; Accepted: October 24, 2017; Published: December 20, 2017

\begin{abstract}
This paper presents a new mathematical model for formation as well as computation of geometric series and summability in step-by-step procedures. Also, it provides mathematical structures for geometric series-ordered terms. The novel mathematical model uses Annamalai's computing method of geometric series and summability, which provided a technique to establish the algorithmic geometric series and its formulae in an earlier paper, for further improvement in the scientific research study. This mathematical/computational models of geometric series are widely used in the fields of physics, engineering, biology, medicine, economics, computer science, queueing theory, finance, and management for the purpose of research and development meeting today's challenges. In an earlier research article, the geometric series along with exponential decay model were used to determine effective medicine dosage. Few specific mathematical formulae had also been discovered by using Annamalai's algorithmic geometric series and summability. This could be very interesting and informative for current students and researchers.
\end{abstract}

Keywords: Algorithmic Geometric Series, Mathematical Structure, Annamalai’s Computing Model, Summability

\section{Introduction}

Geometric series [1-10] played a vital role in differential and integral calculus at the earlier stage of development and still continues as an important part of the study in science, mathematics, economics, management and its applications [3-6]. This paper introduces a practical approach for developing a new mathematical/computational model called Annamalai's algorithmic geometric series and summability and also provides various detailed mathematical structures and new geometric series-formulae such as $\sum_{i=k}^{\infty} a y^{i}=\frac{a y^{k}}{1-y}$, $\sum_{i=0}^{\infty} \sum_{j=i}^{\infty} a y^{j}=\frac{a}{(1-y)^{2}} \quad, \quad \sum_{i=1}^{\infty} \sum_{j=i}^{\infty} a y^{j}=\frac{a y}{(1-y)^{2}} \quad, \quad$ and $\sum_{i=k}^{\infty} \sum_{j=i}^{\infty} a y^{j}=\frac{a y^{k}}{(1-y)^{2}}, \quad(0<y<1)$. These algorithmic geometric series and its formulae could be used in the research areas of biology, medicine, physical science, econometrics, statistics, finance, and management for further development of research techniques and/or technologies meeting today's challenges. The formation and computation of Annamalai's algorithmic geometric series and summability differ from old pattern of geometric series and its mathematical expression. These novel mathematical techniques, methods, and models could be very interesting and informative for currents research scholars to enhance their knowledge and skills further.

\subsection{Geometric Series}

We commonly know that a geometric series is any series that can be written in the following form along with short notation:

$$
a+a r+a r^{2}+a r^{3}+\ldots \ldots . . \text { OR } \sum_{i=0}^{\infty} a r^{i}, \text { where } \mathrm{r} \text { is the }
$$

common ration between any two successive terms.

Summations of finite and infinite geometric series and its formulae were

$$
\sum_{i=0}^{n-1} a x^{i}=\frac{a\left(x^{n}-1\right)}{(x-1)} \text { and } \sum_{i=1}^{n-1} a x^{i}=\frac{a\left(x^{n}-x\right)}{x-1}, x \neq 1
$$




$$
\sum_{i=0}^{\infty} a x^{i}=\frac{a}{1-x} \text { and } \sum_{i=1}^{\infty} a x^{i}=\frac{a x}{1-x}(0<x<1)
$$

\subsection{Decimal to Fraction}

Geometric series can be used to convert the decimal to a fraction.

For examples,

(i) $0.9999999 \ldots \ldots=\frac{9}{10}+\frac{9}{10^{2}}+\frac{9}{10^{3}}+\ldots \ldots . .=\frac{a x}{1-x}=1$ where $a=9$ and $x=\frac{1}{10}$

(ii) $9.9999999 \ldots \ldots=9+\frac{9}{10}+\frac{9}{10^{2}}+\frac{9}{10^{3}}+\ldots \ldots .=\frac{a}{1-x}=10$ where $a=9$ and $x=\frac{1}{10}$

(iii) $0.777777 \ldots \ldots \ldots=\frac{7}{10}+\frac{7}{10^{2}}+\frac{7}{10^{3}}+\ldots . .=\frac{a x}{1-x}=\frac{7}{9}$ where $a=7$ and $x=\frac{1}{10}$

\section{Annamalai's Algorithmic Geometric Series}

First, the great idea [1] for developing the algorithmic geometric series generated from the mathematical equality such as $2=$ 2.

i.e., $2=2 \Rightarrow 2=1+1 \Rightarrow 2=1+\frac{1}{2}+\frac{1}{2} \Rightarrow 2=1+\frac{1}{2}+\frac{1}{4}+\frac{1}{4} \Rightarrow 2=1+\frac{1}{2}+\frac{1}{2^{2}}+\frac{1}{2^{3}}+\frac{1}{2^{3}}$

Similarly, it can be extended the above mathematical expression as under:

$\Rightarrow 2=1+\frac{1}{2}+\frac{1}{2^{2}}+\frac{1}{2^{3}}+\frac{1}{2^{4}}+\ldots \ldots . .+\frac{1}{2^{i}}$, where $1=1,2,3,4, \ldots . ., n$

i.e., $\Rightarrow 2=1+\frac{1}{2}+\frac{1}{2^{2}}+\frac{1}{2^{3}}+\frac{1}{2^{4}}+\ldots \ldots . .+\frac{1}{2^{n-1}}+\frac{1}{2^{n}}+\frac{1}{2^{n}}$

Now it is concluded that $1+\frac{1}{2}+\frac{1}{2^{2}}+\frac{1}{2^{3}}+\frac{1}{2^{4}}+\ldots \ldots . .+\frac{1}{2^{n-1}}+\frac{1}{2^{n}}+=2-\frac{1}{2^{n}}$

Next, the new geometric series [2] is derived from the equality $2^{n}=2^{n}$

$$
\begin{aligned}
& 2^{n}=2^{n-1}+2^{n-1} \Rightarrow 2^{n}=2^{n-1}+2^{n-2}+2^{n-3} \ldots .+2^{2}+2+1+1 \Rightarrow \sum_{i=0}^{n-1} 2^{i}=2^{n}-1 \text { OR } \\
& 2^{n}=2^{n-1}+2^{n-1} \Rightarrow 2^{n}=2^{n-1}+2^{n-2}+2^{n-3} \ldots .+2^{k+1}+2^{k}+2^{k} \Rightarrow \sum_{i=k}^{n-1} 2^{i}=2^{n}-2^{k} \text {, where } n \text { and } k \text { are integers with condition }
\end{aligned}
$$

\section{$k \leq n$ OR}

$$
2^{n}=2^{n-1}+2^{n-1} \Rightarrow 2^{n}=2^{n-1}+2^{n-2}+\ldots .2+1+2^{-1}+\ldots . .+2^{-n} \Rightarrow \sum_{i=-n}^{n-1} 2^{i}=2^{n}-2^{-n}, \text { where } n \text { and } k \text { are integers with }
$$

condition $k \leq n$.

Similarly, it can be generated the algorithmic geometric for equality with the integer 100 .

$$
100^{n}=100^{n} \Rightarrow 100^{n}=(100-1) 100^{n-1}+100^{n-1} \Rightarrow 100^{n}=(100-1) 100^{n-1}+(100-1) 100^{n-2}+100^{n-2}
$$

We can further expand the terms as follows:

$$
100^{n}=100^{n} \Rightarrow 100^{n}=(100-1) 100^{n-1}+(100-1) 100^{n-2}+(100-1) 100^{n-3}+\ldots . .+(100-1) 100^{k}+100^{k}
$$

By simplifying we get

$$
100^{n}=100^{n} \Rightarrow \sum_{i=k}^{n-1} 100^{i}=\frac{100^{n}-100^{k}}{100-1}=\frac{100^{n}-100^{k}}{99}
$$

If the integer ' $\mathrm{k}$ ' reaches to the integer ' 0 ', then $100^{n}=100^{n} \Leftrightarrow \sum_{i=0}^{n-1} 100^{i}=\frac{100^{n}-1}{99}$ 


\section{Effective Medicine Dosage Using Geometric Series}

It is understood that for almost all drugs there is a maximum concentration above which the drug is dangerous $[3,4]$, and there is a minimum concentration below which the drug is ineffective. Thus, the physician is compulsorily to give the dosage between minimum and maximum concentrations to a patient for effectiveness [3, 4]; otherwise it can be risky. Let us consider a patient is given the same dose of a medicine at equally spaced time intervals. The dose concentration in the bloodstream decreases as the drug is broken down by the body. However, it does not disappear completely before the next dose is given. Let us understand the exponential decay model for the concentration of a drug in a patient's bloodstream.

The function $V(t)$ is used to represent a dose concentration at time $\mathrm{t}$ and $V_{0}$ to represent the concentration just after the dose is administered intravenously. Then the exponential decay model is formulated $[3,4]$ by

$$
V(t)=V_{0} e^{-p t}
$$

where $p$ is the property of the particular drug being used.

Now, let us consider that $V(t)$ be the first dose concentration at time $\mathrm{t}$ and that $V_{0}$ the concentration at time $\mathrm{t}$ $=0$ just after the first dose is administered intravenously. Suppose that at $\mathrm{t}=\mathrm{c}$, a second dose of the drug is given to the patient. The concentration of the drug in the bloodstream jumps almost immediately to its highest level $V(c)$ and then the concentration is diffused so rapidly throughout the bloodstream over time. The expression $V(t)=V_{0} e^{-p t}$ is valid as long as only a single dose is given.

During the time between the second and third doses, the concentration decays exponentially from this value. To find the concentration after the third dose, the same process must be repeated.

At $\mathrm{t}=\mathrm{c}$, the dose concentration is calculated [3, 4] as $V\left(c^{-}\right)=V_{0} e^{-p c}$ just before the second dose is administered intravenously.

Here, $V\left(c^{-}\right)=\lim _{t \rightarrow c^{-}} V(t)$.

When the second dose is administered intravenously, the concentration jumps by an increment $V_{0}$, i.e. the concentration just after the second dose given is

$$
V\left(c^{+}\right)=V_{0}+V\left(c^{-}\right)=V_{0}+V_{0} e^{-p c}=V_{0}\left(1+e^{-p c}\right) .
$$

Note that $V\left(c^{-}\right)$denotes 'just before the new dose is administered' and $V\left(c^{+}\right)$denotes 'just after the new dose is administered'.

The concentration then decays from this value according to the exponential decay rule, but with a slight twist. The twist is that the initial concentration is at $\mathrm{t}=\mathrm{c}$, instead of $\mathrm{t}=0$. One way to handle this is to write the exponential term as $e^{-p(t-c)}$ so that at $\mathrm{t}=\mathrm{c}$, the exponent is 0 . If we do this, then we can write the concentration as a function of time as

$$
V(t)=V_{0}\left(1+e^{-p c}\right) e^{-p(t-c)}
$$

This function is only valid after the second dose is administered and before the third dose is given. That is, for $c \leq t<2 c$.

Now, suppose that a third dose of the drug is given at $\mathrm{t}=$ 2c. The concentration just before the third dose is given would be $V\left(2 c^{-}\right)$, which is

$$
V\left(2 c^{-}\right)=V\left(c^{+}\right) e^{-p c}=V_{0}\left(1+e^{-p c}\right) e^{-p c}
$$

i.e.,

$$
V\left(2 c^{-}\right)=V_{0}\left(e^{-p c}+e^{-2 p c}\right)
$$

When the third dose is given, the concentration would jump again by $V_{0}$ and the concentration just after the third dose would be

$$
V\left(2 c^{+}\right)=V_{0}+V\left(2 c^{-}\right)=V_{0}\left(1+e^{-p c}+e^{-2 p c}\right)
$$

Now, suppose that a forth dose of the drug is given at $\mathrm{t}=$ $3 \mathrm{c}$. The concentration just before the forth dose is given would be $V\left(3 c^{-}\right)$, which is

$$
V\left(3 c^{-}\right)=V(2 c+) e^{-p c}=V_{0}\left(e^{-p c}+e^{-2 p c}+e^{-3 p c}\right)
$$

When the third dose is given, the concentration would jump again by $V_{0}$ and the concentration just after the third dose would be

$$
V\left(3 c^{+}\right)=V_{0}+V\left(3 c^{-}\right)=V_{0}\left(1+e^{-p c}+e^{-2 p c}+e^{-3 p c}\right)
$$

The concentration just before the $n$-th dose of the drug would be

$$
V\left((n-1) c^{-}\right)=V_{0} \sum_{j=1}^{n-1} e^{-j p c}
$$

The concentration just after the $n$-th dose of the drug would be

$$
V\left((n-1) c^{+}\right)=V_{0} \sum_{j=0}^{n-1} e^{-j p c}
$$

Let $u=e^{-p c}$

Note that $0<u<1$, since $\mathrm{k}$ and $\mathrm{c}$ are both positive constants.

From the geometric series (1) and (2), we formulate as

$$
V\left((n-1) c^{-}\right)=V_{0} \sum_{j=1}^{n-1} u^{j}=V_{0}\left(\frac{u-u^{n}}{1-u}\right)
$$


and

$$
V\left((n-1) c^{+}\right)=V_{0}+V\left((n-1) c^{-}\right)=V_{0} \sum_{j=0}^{n-1} u^{j}=V_{0}\left(\frac{1-u^{n}}{1-u}\right)
$$

The equations (3) and (4) are formulae for the partial sum of a geometric series.

Suppose a treatment for a patient is continued indefinitely. Then the equation (4) becomes

$$
V\left((n-1) c^{+}\right)=\lim _{n \rightarrow \infty} V_{0}\left(\frac{1-u^{n}}{1-u}\right)=V_{0}\left(\frac{1}{1-u}\right)
$$

Now, we conclude from the results that the minimum concentration is the concentration just before the second dose is given,

$$
\sum_{i=-m}^{n-1} a x^{i}=\frac{a\left(x^{n}-x^{-m}\right)}{x-1} \Leftrightarrow a x^{n}=a x^{n},(x \neq 1)
$$

Proof

$$
\begin{gathered}
\text { RHS } \Rightarrow a x^{n}=a x^{n} \\
\Rightarrow a x^{n}=a(x-1) x^{n-1}+a x^{n-1} \\
\Rightarrow a x^{n}=a(x-1) x^{n-1}+a(x-1) x^{n-2}+\ldots+a(x-1) x^{i}+\ldots .+a(x-1) x^{-m}+a x^{-m} \\
a x^{n}=a x^{n} \Rightarrow \sum_{i=-m}^{n-1} a x^{i}=\frac{a\left(x^{n}-x^{-m}\right)}{x-1} \\
\text { LHS } \Rightarrow \sum_{i=-m}^{n-1} a x^{i}=\frac{a\left(x^{n}-x^{-m}\right)}{x-1} \\
\Rightarrow a(x-1) x^{n-1}+a(x-1) x^{n-2}+\ldots+a(x-1) x^{i}+\ldots .+a(x-1) x^{-m}+a x^{-m} \\
\sum_{i=-m}^{n-1} a x^{i}=\frac{a\left(x^{n}-x^{-m}\right)}{x-1} \Rightarrow a x^{n}=a x^{n}
\end{gathered}
$$

From (1) and (2) we get:

$$
\sum_{i=-m}^{n-1} a x^{i}=\frac{a\left(x^{n}-x^{-m}\right)}{x-1} \Leftrightarrow a x^{n}=a x^{n},(x \neq 1)
$$

From the above result we can further find the following summability:

(i) $a \sum_{i=k}^{n-1} x^{i}=\frac{a\left(x^{n}-1+1-x^{k}\right)}{x-1}=\frac{a\left(x^{n}-1\right)}{x-1}-\frac{a\left(x^{k}-1\right)}{x-1}=a\left(\sum_{i=0}^{n-1} x^{i}-\sum_{i=0}^{k-1} x^{i}\right)$ 
(ii) $\sum_{i=-m}^{n-1} a x^{i}=\sum_{i=-m}^{-1} a x^{i}+\sum_{i=0}^{n-1} a x^{i}=\sum_{i=1}^{m} \frac{a}{x^{i}}+\sum_{i=o}^{n-1} a x^{i}=a\left(\frac{\frac{1}{x}-\frac{1}{x^{m+1}}}{1-\frac{1}{x}}+\frac{x^{n}-1}{x-1}\right)=\frac{a\left(x^{n}-x^{-m}\right)}{x-1}$

Now, the equality $a x=a x$ was used to design the mathematical/computational modelling,

$$
a x=a x \Leftrightarrow a x=(x-1) a+a \Leftrightarrow a x=(x-1) \frac{a}{x^{0}}+(x-1) \frac{a}{x}+(x-1) \frac{a}{x^{2}}+\ldots .+(x-1) \frac{a}{x^{n}}+\frac{a}{x^{n}}
$$

$a x=a x \Leftrightarrow \sum_{1=0}^{n} \frac{a}{x^{i}}=\frac{\left(a x-\frac{a}{x^{n}}\right)}{x-1}=\frac{a x\left(1-\frac{1}{x^{n+1}}\right)}{x\left(1-\frac{1}{x}\right)}=\frac{a\left(1-y^{n+1}\right)}{1-y}$ where $y=\frac{1}{x}$

Now $\frac{a}{y}=\frac{a}{y} \Leftrightarrow \sum_{i=0}^{n-1} a y^{i}=\frac{a\left(y^{n}-1\right)}{y-1}$ where it is understood that $\frac{a}{y}=\frac{a}{y} \Rightarrow a y=a y$

We know that if $0<y<1$, then $\sum_{i=0}^{n-1} a y^{i}=\frac{a\left(1-y^{n}\right)}{1-y}$ and $\sum_{i=0}^{\alpha} a y^{i}=\frac{a}{1-y}$

Similarly, using Annamalai computing geometric series $a \sum_{i=k}^{n-1} y^{i}=\frac{a\left(y^{n}-y^{k}\right)}{y-1} \quad(y \neq 1)$,

we can derive $a \sum_{i=k}^{n-1} y^{i}=\frac{a\left(y^{k}-y^{n}\right)}{1-y}$ and $a \sum_{i=k}^{\alpha} y^{i}=\frac{a y^{k}}{1-y}(0<y<1)$

where $k>0$ is an integer constant.

From the above result it can further found the following summability:

(i) $\sum_{i=0}^{\infty} a y^{i}-\sum_{i=k}^{\infty} a y^{i}=\frac{a}{1-y}-\frac{a y^{k}}{1-y}=\frac{a\left(1-y^{k}\right)}{1-y}=\sum_{i=0}^{k-1} a y^{i}$

(ii) $\sum_{i=0}^{\infty} \sum_{j=i}^{\infty} a y^{j}=\sum_{j=0}^{\infty} a y^{j}+\sum_{j=1}^{\infty} a y^{j}+\sum_{j=2}^{\infty} a y^{j}+\ldots . .=\frac{a}{1-y}+\frac{a y}{1-y}+\frac{a y^{2}}{i-y}+\ldots . .=\frac{a}{(1-y)^{2}}$

(iii) $\sum_{i=1}^{\infty} \sum_{j=i}^{\infty} a y^{j}=\sum_{j=0}^{\infty} a y^{j}+\sum_{j=1}^{\infty} a y^{j}+\sum_{j=2}^{\infty} a y^{j}+\ldots . .=\frac{a y}{1-y}+\frac{a y}{1-y}+\frac{a y^{2}}{i-y}+\ldots . .=\frac{a y}{(1-y)^{2}}$

(iv) $\sum_{i=k}^{\infty} \sum_{j=i}^{\infty} a y^{j}=\sum_{j=0}^{\infty} a y^{j}+\sum_{j=1}^{\infty} a y^{j}+\sum_{j=2}^{\infty} a y^{j}+\ldots . .=\frac{a y^{k}}{1-y}+\frac{a y^{k+1}}{1-y}+\frac{a y^{k+2}}{i-y}+\ldots . .=\frac{a y^{k}}{(1-y)^{2}}$

\section{Mathematical Structures}

Mathematical structure for a general term in geometric series is:

$$
\sum_{i=n}^{n} a x^{i}=\frac{a\left(x^{n+1}-x^{n}\right)}{x-1}=\frac{a x^{n}(x-1)}{x-1}=a x^{n}
$$

Using the above research idea we can constitute mathematical structures in theoretical aspects of computing for each term in the geometric series $1+x+x^{2}+x^{3}+\ldots+x^{k}$.

They are:

$$
1=\frac{x-1}{x-1} ; x=\frac{x^{2}-x}{x-1} ; x^{2}=\frac{x^{3}-x^{2}}{x-1} ; x^{3}=\frac{x^{4}-x^{3}}{x-1}
$$

$$
x^{4}=\frac{x^{5}-x^{4}}{x-1} ; \ldots \ldots . . ; x^{k-1}=\frac{x^{k}-x^{k-1}}{x-1} ; x^{k}=\frac{x^{k+1}-x^{k}}{x-1}
$$

Similarly, mathematical structures for 2-term geometric series are:

$$
\sum_{i=n}^{n+1} a x^{i}=\frac{a\left(x^{n+2}-x^{n}\right)}{x-1}=a x^{n+1}+a x^{n}
$$

$$
1+x=\frac{x^{2}-1}{x-1} ; x+x^{2}=\frac{x^{3}-x}{x-1} ; x^{2}+x^{3}=\frac{x^{4}-x^{2}}{x-1} ; \ldots \ldots ;
$$

$x^{n}+x^{n-1}=\frac{x^{n+1}-x^{n-1}}{x-1}$

Also, mathematical structures for 3-term geometric series 
are:

$$
\begin{gathered}
\sum_{i=n}^{n+2} a x^{i}=\frac{a\left(x^{n+3}-x^{n}\right)}{x-1}=a x^{n+2}+a x^{n+1}+a x^{n} \\
1+x+x^{2}=\frac{x^{3}-1}{x-1} ; x+x^{2}+x^{3}=\frac{x^{4}-x}{x-1} ; x^{2}+x^{3}+x^{4}=\frac{x^{5}-x^{2}}{x-1} ;
\end{gathered}
$$
and so on.

For i-term geometric series the mathematical structures are built as the above-mentioned structures where ' $i$ ' is a set of integers $\{-\propto, \ldots .-3,-2,-1,0,1,2,3, \ldots .+\propto\}$.

For computing of n-term geometric series the mathematical structures are built as follows:

$$
\sum_{i=t}^{t+(n-1)} a x^{i}=\frac{a\left(x^{t+n}-x^{t}\right)}{x-1}=a x^{t+(n-1)}+\ldots+a x^{t+1}+a x^{t}
$$

OR

$$
x^{t}+x^{t+1}+x^{t+2}+\ldots+. x^{t+(n-1)}=\frac{x^{t+n}-x^{t}}{x-1}
$$

For infinite geometric series, we can make the mathematical structure as follows:

$$
\sum_{i=k}^{\infty} a x^{i}=\frac{a x^{k}}{x-1} \text { OR } \sum_{i=k}^{\infty} x^{i}=\frac{x^{k}}{x-1}
$$

\section{Conclusion}

In this paper, a new mathematical/computational model for Annamalai's algorithmic geometric series and summability has been discovered for research purpose in the fields of physics, engineering, biology, medicine, economics, computer science, queueing theory, finance, and management

Also, we have developed the mathematical structures for geometric series-ordered terms in novel ways. The novel computational model uses Annamalai's computing method of geometric series and summability, which provided a technique to establish the algorithmic geometric series and its formulae in an earlier paper, for further improvement in the scientific research study. In an earlier research article, the geometric series along with exponential decay model were used to determine effective medicine dosage. The newly developed computational models are:

$$
\begin{gathered}
\sum_{i=k}^{\infty} a y^{i}=\frac{a y^{k}}{1-y}, \sum_{i=0}^{\infty} \sum_{j=i}^{\infty} a y^{j}=\frac{a}{(1-y)^{2}}, \\
\sum_{i=1}^{\infty} \sum_{j=i}^{\infty} a y^{j}=\frac{a y}{(1-y)^{2}}, \text { and } \sum_{i=k}^{\infty} \sum_{j=i}^{\infty} a y^{j}=\frac{a y^{k}}{(1-y)^{2}},(0<y<1) .
\end{gathered}
$$

These mathematical structures and models can be widely used further for research and development meeting today's challenges.

\section{References}

[1] Annamalai C 2009 "Computational geometric series model with key applications in informatics", International Journal of Computational Intelligence Research, Vol 5(4), pp 485-499.

[2] Annamalai C 2009 "A novel computational technique for the geometric progression of powers of two", Journal of Scientific and Mathematical Research, Vol 3, pp 16-17.

[3] Annamalai C 2010 "Applications of Exponential Decay and Geometric Series in Effective Medicine Dosage", Journal Advances in Bioscience and Biotechnology, Vol 1, pp 51-54.

[4] Annamalai C 2011 "Computational Model to study the Dose Concentration in Bloodstream of Patients", International Journal of Medical and Pharmaceutical Sciences, Vol 1(5), pp $1-7$.

[5] Annamalai C 2011 "ACM cryptographic key exchange for secure communications", International Journal of Cryptology Research, Vol. 3(1), pp 27-33.

[6] Ramya R 2015 "Geometric Series in Financial Mathematics", International Journal of Multidisciplinary Research and Modern Education, Vol 1(1), pp 305-308.

[7] Annamalai C 2015 "A Novel Approach to ACM-Geometric Progression", Journal of Basic and Applied Research International, Vol. 2(1), pp 39-40.

[8] Annamalai C 2017 "Annamalai Computing Method for Formation of Geometric Series using in Science and Technology, International Journal for Science and Advance Research in Technology, Vol. 3(8), pp 287-289.

[9] Donna Roberts, Geometric Sequences and Series.

[10] Alfred S. Posamentier, Charles T. Salkind, Challenging Problems in Algebra.

\section{Biography}

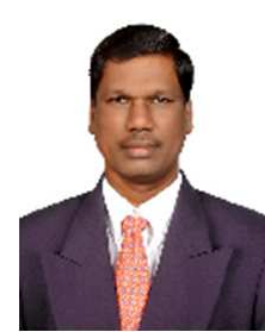

Chinnaraji Annamalai has experience for more than 20 years in research and teaching of computer science and information technology and admin. He has published several papers in diverse fields of science and technology. Also, he has reviewed recent IT and Computer books published by William Stallings (USA) and many other articles published by IEEE journals and top journals in the fields of computing sciences and its related subjects. Presently he is working at Indian Institute of Technology Kharagpur. 